\title{
Quelques réflexions sur les curriculums Canada Dry ${ }^{\circledR}$
}

\author{
A few thoughts concerning Canada Dry curricula
}

Jean-Jacques GUILBERT

Résumé Propos : Le lecteur se souvient peut-être d'une publicité pour une boisson qui, nous disait la firme Canada Dry ${ }^{\circledR}$, avait " la couleur de l'alcool, le goût de l'alcool, mais qui n’était pas de l'alcool ». En éducation médicale il est de nos jours de bon ton de s'habiller à la mode et de mettre l'accent pédagogique sur des notions telles que "l'orientation communautaire ", "l'apprenant au centre du dispositif de formation" ou encore "le processus par résolution de problèmes", alors que dans la réalité les "nouveaux enseignements " annoncés restent pratiquement sans changement par rapport au passé. Méthode: L'auteur utilise la méthode de recherche par analyse sémantique de contenu de textes. Messages clés: Le présent article fait des propositions prédictives sur les chances de réussite de plans de réformes récents. Les réformes annoncées permettent d'aborder d'une manière générale un sujet sensible et complexe. Conclusion: L'auteur recommande notamment de focaliser une réforme sur l'évaluation des compétences professionnelles acquises par les étudiants plutôt que sur la forme de l'enseignement ou tout autre élément de la spirale de planification de l'éducation.

Mots clés Analyse sémantique de contenu ; apprentissage ; compétences professionnelles ; démocratie ; enseignement ; études médicales ; évaluation formative ; examens de certification ; objectifs pédagogiques ; plan de réforme ; validité.

Abstract Purpose: The reader may remember a television commercial from the firm Canada Dry, promoting a soft drink saying that it "had the color of alcohol, the taste of alcohol, but did not contain alcohol". In today's medical education, it is politically correct to be "learner centered", "community oriented" and "problem-based" but "new programs" remain practically identical to older ones. Method: The author used semantic analysis research based method. Key messages: The paper reveals predictive proposals regarding the chances of succeeding such "new programs". Conclusion: The author recommendations focus on a reform that prompts assessment of acquired professional performances by learners rather than assessment on teaching methods or on any other traditional pedagogical basics.

Key words Semantic analysis; learning;, professional competencies; democracy; teaching, medical education; formative assessment; certifying evaluation;, educational objective;, reform plans; validity.

Pédagogie Médicale 2006; 7: 53-8

Correspondance: J-J. Guilbert, Professeur (ret.), 15 avenue du Mail. CH - 1205 Genève -Téléphone : + 41 (0)22 3202997 Mailto:guilbertjj@yahoo.fr 


\section{Note liminaire}

Sauf erreur, toute ressemblance avec des citations déjà publiées ne peut être que le réjouissant effet d'une coïncidence basée sur cinquante années d'expérience du milieu hospitalo-universitaire. L'auteur utilise la méthode de recherche par analyse sémantique de contenu de textes en utilisant comme référence comparative un ouvrage personnel précédemment publié . Il rend compte de sa perception de ce qui sous-tend la planification de réformes annoncées. Cet article est l'occasion de montrer une fois de plus combien sont grandes les difficultés rencontrées à l'occasion de réformes des études médicales ${ }^{2-6}$. Le but n'est pas de faire la leçon à des collègues inconnus qui, bien entendu, gardent tout son respect confraternel. Certains lecteurs, minoritaires, ne reconnaîtront pas leur milieu habituel. Les autres sont confraternellement invités à réfléchir et à éventuellement proposer une vision alternative.

\section{Introduction}

Le lecteur se souvient peut-être d'une publicité pour une boisson qui, nous disait la firme Canada Dry ${ }^{\circledR}$, avait « la couleur de l'alcool, le goût de l'alcool, mais qui n'était pas de l'alcool ». En éducation médicale il est de nos jours de bon ton de s'habiller à la mode et de mettre l'accent pédagogique sur des notions telles que « l'orientation communautaire ", « l'apprenant au centre du dispositif de formation" ou encore "le processus par résolution de problèmes ", alors que, dans la réalité, les "nouveaux enseignements " annoncés restent pratiquement sans changement par rapport au passé.

\section{Une louable intention souvent déclarée : "Former les médecins de demain vers les soins aux malades"}

Hors de cette lapalissade, il est de bon ton de mentionner d'emblée qu'un nouveau programme de médecine " oriente ses activités vers les soins aux malades " même si le lecteur lambda a peu de risque de ne pas l'imaginer tout seul. Mais l'annonce d'une réforme est souvent présentée, inter alia, comme une réponse soit au développement des sciences fondamentales, soit à celui des techniques d'investigation et de thérapeutique cliniques et pratiquement jamais à une demande de la population.

Il y est dit que le médecin de demain doit avoir, au terme du cursus de formation, des compétences professionnelles que l'on imagine a priori au bénéfice des patients : « compétences relationnelles " ou "savoir être ", "savoir-faire " ou " gestes pratiques » et - comme toujours - du " savoir " qui correspond presque toujours d'avantage à des connaissances contributives à mémoriser pour le jour de l'examen qu'aux « compétences intellectuelles » indispensables pour faire un diagnostic et décider d'une thérapie.

Lorsqu'ils existent, il est fait référence à des « objectifs d'apprentissage ». Trop souvent ce sont des objectifs disciplinaires ( «nommer les 7 branches du nerf radial») au lieu de décrire des compétences professionnelles ("diagnostiquer par l'examen physique un déficit du nerf radial »). On nous explique le pourquoi et le comment de la relation quasi consanguine entre la biologie (les sciences dites fondamentales) et la médecine, sans se perdre toutefois à expliquer pourquoi, dans la réalité des programmes, ces deux éléments (clinique et préclinique) sont séparés, consécutifs et très souvent sans liens entre eux.

\section{On a le souci de maintenir et développer la qualité de l'enseignement}

Il est précisé que pour parvenir à maitriser les compétences professionnelles « les étudiant [doivent] se former par euxmêmes dans le cadre de leurs études et durant toute leur carrière ". On attire ainsi l'attention sur les « nouvelles approches pédagogiques favorisant l'apprentissage en petits groupes plutôt que l'enseignement ex-cathedra ». Le qualificatif de nouveauté concernant une approche déjà utilisée au Moyen Age et remise à la mode il y a près de 40 ans à McMaster (Canada) mérite réflexion. De plus, la promesse est toute relative car l'analyse des horaires d'enseignement montre la bonne place encore réservée à l'enseignement ex-cathedra. "L'étudiant continue à rester passif... avec une forme prépondérante d'enseignement ex-cathedra $"$.

Il existe de plus en plus souvent une unité de pédagogie médicale. Il y est de bon ton de mentionner que les "objectifs d'apprentissage [sont] devenus une pierre angulaire de l'enseignement médical ". Il est aussi moderne de se référer au concept d'assurance qualité (Type ISO 9000), même s'il est admis que le concept ISO s'appliquerait difficilement pour vérifier la qualité de l'enseignement car « il n'est pas possible de contrôler tout ce qui influence un étudiant entre son entrée à l'université et la fin de son cursus ». Un promoteur du concept ISO considère ${ }^{8}$ que la " qualité » serait " une régulation des tensions entre des besoins et des priorités différentes ", à savoir les attentes et besoins des étudiants (la réussite aux examens) et les priorités des enseignants (un apprentissage profond ${ }^{9}$ ou une appropriation durable des contenus). De tous temps la " réussite aux examens" a été le 
moteur principal de l'action. Ne serait-il pas alors temps de contrôler avec un meilleur niveau de validité la " qualité ISO » des diplômés en vérifiant la performance de compétences professionnelles ${ }^{10}$ ?

\section{Un nouveau curriculum pour mieux répondre aux besoins des malades et de la société}

On entre là en pleine pertinence sociétale. C'est au Conseil de l'Ecole de médecine - ou autre appellation - de formuler « les lignes directrices » indiquant que le nouveau curriculum serait : «basé sur des objectifs à atteindre » de " compétences cliniques et relationnelles avec les malades ", " adapté aux futures activités professionnelles » des étudiants, "lié à des besoins spécifiques de la population »; basé sur " des connaissances approfondies dans les sciences fondamentales tout au long des études ", selon " un apprentissage progressif allant du simple au compliqué » (évidence fausse, familière et incontournable, qui va à l'encontre de la pratique professionnelle qui part obligatoirement du patient plus souvent " compliqué " que "simple ») « dans une attitude participative [...] d'indépendance intellectuelle développant une aptitude à résoudre des cas concrets dans leur complexité individuelle " ${ }^{11}$. C'est beau comme de l'Antique.

Le «calendrier » schématisant un " nouveau curriculum " décrit en général "trois phases ». Exemple classique, la première phase comprend deux semestres « de sciences fondamentales » dans " une approche intégrée de l'enseignement " avec des " modules autonomes " donnant droit à des crédits, en conformité avec le European Credit Transfer System de la Convention de Bologne. Cette phase est suivie de celle " de clinique propédeutique " puis de la phase "d'immersion clinique " avec mention de "résolution de problèmes cliniques ». Les "lignes directrices" sont politiquement correctes mais le " calendrier " reste trop souvent d'apparence classique. Serait-ce du pur Canada Dry ${ }^{\circledR}$ ?

La première phase, dite nouvelle, abandonnerait le monopole classique des conférences magistrales par disciplines cloisonnées et adopterait l'approche dite de l' "enseignement intégré ". Parée de la magie du mot " intégré ", elle permet d'espérer une amélioration par rapport à la réalité des programmes classiques où chaque discipline menait sa propre vie. Or l'autonomie traditionnelle des disciplines est reconnue comme un facteur inhibant un processus de changement. Malheureusement la littérature montre que ce choix pédagogique de l'ensei- gnement intégré, a toujours mené à l'échec ${ }^{12}$. Utilisée pour la première fois, en 1951, à Western Reserve (Cleveland, Etat-Unis) cette approche a été abandonnée avec fracas par Harvard et Yale en 1971. Mais, à part au Québec, elle est encore utilisée un demi-siècle plus tard dans beaucoup d'écoles de médecine francophones. Son application requiert de la part des enseignants un effort considérable, soutenu et fort dispendieux en temps. Ils digèrent collectivement les " connaissances indispensables » pour nourrir les étudiants alors qu'il serait plus efficace d'amener les étudiants à développer leurs méthodes de travail pour trouver leur nourriture pendant et après leur formation de base. McMaster (Canada), dès 1967, en a fait une démonstration convaincante par un "apprentissage intégrant » selon l'approche par résolution de problèmes. Environ $10 \%$ seulement des écoles de médecine y sont aussi parvenues. La critique est facile, mais passer d'une mentalité collective " centrée sur l'enseignement " à l'adoption d'une approche "centrée sur l'apprentissage " est un saut psycho-intellectuel géant. Kerr L. White a montré que "It takes 50 years for a new idea to catch on " ${ }^{13}$. Le seul "bon coté » de l'enseignement intégré est d'être en apparence à l'avantage des étudiants. Ils n'ont plus à faire l'effort de rechercher le contenu utile à mémoriser pour le jour des examens, ni celui de le digérer, car il a été prédigéré par les enseignants. Les étudiants peuvent juste se préparer à régurgiter le tout au moment des examens. L'aptitude à mémoriser reste prépondérante mais avec un résultat éphémère tout en laissant dans le domaine du rêve l'aptitude à résoudre des problèmes et soigner une hypertrophie curriculaire ${ }^{14}$ due au développement des sciences et de la technologie.

Reste à mentionner le manque d'intégration des deux volets complémentaires de la formation, clinique et préclinique. Cette classique séparation, arbitraire et néfaste ${ }^{13}$, persiste malheureusement pratiquement partout.

La description de la deuxième phase exprime l'intention d'aller plus loin qu' " une journée dans le cabinet d'un praticien en groupe de deux étudiants " mais il faudra se contenter d'un " cours de révision en petit groupe [...] unique contact avec un patient durant la sixième année " car " chez les étudiants finalistes ", " on a constaté des difficultés récurrentes dans l'application des compétences cliniques de base ». Eh oui, ça arrive ! Et pendant ce temps là, les collègues de médecine générale font le "rêve » émouvant et éloigné du pur Canada Dry ${ }^{\circledR}$ « d'un curriculum qui ne partira plus d'une discipline mais d'un patient, d'un problème concret qui imposera [...] une approche holistique ». Un beau rêve tout à fait cohérent avec les « lignes directrices » présentées plus avant « au 
nom du Conseil de l'Ecole de médecine ». Mais passer du rêve à la réalité semble bien être un autre rêve.

\section{Les étudiants participent à l'établissement d'un nouveau curriculum}

Il est moderne de pouvoir dire que les étudiants jouent un rôle et que l'on tient compte de leurs opinions. On leur donne la parole pour qu'ils expriment un fort sentiment de satisfaction car " tout le monde avait envie de modifier le curriculum » afin de le " médicaliser, surtout pendant les premières années [et] augmenter le contact avec les malades " et rendre l'étudiant " prêt à assumer ses fonctions futures de médecin [...] pour répondre aux attentes des malades et de la société ». Ainsi « l'étudiant devra apprendre à étudier de façon indépendante beaucoup plus que par le passé $»^{15}$. Cet étudiant optimiste fait bien ressortir "le point crucial [de se baser] sur des objectifs d'apprentissage prédéfinis ». Il rassure en disant que « les étudiants ne font pas de la figuration au sein des commissions » et que « leur avis est sollicité, pris en compte, si bien que les travaux avancent sur une base de vrai partenariat ». Dans un cas pas réellement imaginaire, il m’a été possible de calculer que, pour les diverses commissions décisionnelles et sur un total de 195 membres, il y a 13 étudiants soit 6,6\% avec vote à majorité simple. Encore un petit verre de Canada Dry ${ }^{\circledR}$ ?

\section{Les décisions pédagogiques bénéficient d'un contrôle démocratique}

La modernité politiquement correcte exige que le mécanisme décisionnel soit sous le contrôle d'" une mini société ouverte, démocratique » impliquant la garantie de la «fameuse liberté académique [...] et, pour les étudiants, une certaine marge (sic) dans le choix de leur parcours de formation ". "Le contrôle démocratique de l'ensemble est confié au conseil de faculté [...], une sorte de parlement ». Tout va bien, non?

Ceux qui connaissent la réalité de la "démocratie " au sein des universités peuvent avoir un doute, d'autant plus que dans cette mini société démocratique il n'est pas fait mention de la population indigène qui finance la structure par le biais des impôts. Une faculté classique est (selon la caricature d'une forme grave) une mini société comprenant une centaine d'enseignants de disciplines diverses, utilisant leurs titres et ancienneté pour prendre toutes décisions « sur la base de la tradition, de l'intuition ou d'idées personnelles plutôt que sur des résultats de recherche en éducation ", si besoin au nom et à la place des milliers d'autres membres de cette société (étudiants et corps intermédiaire, administratif et technique, etc.) et restant « jalousement attachés aux principes de la « liberté académique » et de l'autonomie des départements pour continuer à pratiquer une politique de splendide isolement à l'égard du système de soins de santé ${ }^{16}$ et de la population.

Une réelle "prise en compte " des avis des étudiants (le peuple), qui pèsent objectivement environ cinq pour cent au moment de votes du conseil de faculté pris à la majorité simple des voix, serait-elle la surprenante indication d'une quasi mutation ayant amené un profond changement d'attitude de la part de professeurs qui ont tendance à penser que « les étudiants doivent accepter l'autorité du professeur ; ils n'ont atteint ni le niveau de maturité ni l'expérience leur permettant de porter un jugement, cela conduirait à une dépréciation de l'enseignement $\|^{17}$ ? La " gestion de cette mini société " avec " contrôle démocratique " est certes reconnue comme " pas facile». Ces adaptations sous forme de "démocratie représentative " ou « constitutionnelle » ne nous font pas oublier les « démocraties populaires » décrites par Orwell. De plus, le processus démocratique est peut-être une fausse bonne solution. Margaret Bussigel apporte des arguments solides pour montrer que l'approche démocratique, d'apparence politiquement correcte, n'est pas forcément un élément positif pour la mise en ouvre d'une réforme ${ }^{12}$. En France une importante réforme au niveau national dite "Réforme Debré " fut tout, sauf " démocratique " car imposée par ordonnance (1959) du président Ch. de Gaulle, sans passer par un vote du parlement. Cette réforme institua, inter alia, le plein temps des professeurs, l'externat pour tous, l'« enseignement intégré » et les examens par questions à choix multiple. Une approche par démocratie directe n'aurait jamais permis de faire des modifications aussi notables. Rien dans la littérature ne permet d'affirmer qu'une approche démocratique, ou son absence, soit en relation directe avec la qualité, ou non, d'une réforme. On sait, en revanche, que l'on ne construit jamais un gratte-ciel ou un pont suspendu en suivant un processus démocratique. Un architecte compétent et déterminé avec une touche de génie s'entoure d'une équipe soudée et va de l'avant. Une faculté de médecine doit avoir toutes les qualités d'une université tout en étant une école professionnelle qui aide à « construire " les médecins de demain

\section{Conclusion en forme de recommandations}

Les recommandations qui suivent découlent de la relation 
entre les intentions politiquement correctes annoncées et les actions prises correspondantes.

La bonne foi, la conscience professionnelle et le désir de bien faire des collègues fictifs auxquels sont attribuées les citations tout autant imaginaires ne peuvent être mis en doute. On peut même supposer qu'ils ont une opinion suffisamment positive de leurs efforts et du succès attendu pour parfois le faire connaître par un texte promotionnel dans une revue professionnelle (dont le coût est d'environ 10000 à $15000 €)$.

On peut se sentir rassuré avec eux quant à l'espoir raisonnable que leurs futures promotions d'étudiants continueront comme par le passé d'obtenir des résultats satisfaisants aux examens de certification. L'expérience internationale et la recherche pédagogique n'ont jamais montré une relation directe et significative entre la " qualité " des programmes d'enseignement avant ou après une « réforme » et le succès des étudiants aux examens de certification, ni encore moins avec les compétences professionnelles acquises. Les examens mesurent plus souvent un exploit de mémorisation que la performance de compétences professionnelles et les étudiants font bien ce qu'il faut pour s'adapter.

S'il est de bon ton de dire que les « objectifs d'apprentissage [sont] devenus une pierre angulaire de l'enseignement médical ", il reste à mieux définir ces objectifs pour permettre de vérifier le gain de compétences professionnelles des étudiants entre leur entrée à l'université et la fin de leur cursus.

Mais le plus important reste que l'évaluation de la performance des étudiants est, classiquement, le chaînon de moindre qualité de la spirale de planification de l'éducation. C'est d'autant plus regrettable que cette faiblesse connue ne permet pas de savoir si les diplômés sont compétents. C'est trop tard que l'on mesure l'étendue des erreurs médicales évitables commises par incompétence professionnelle.

President Bill CLINTON on health care: The Rose Garden. Dec 7, 1999. "According to a disturbing report of the Institute of Medicine about patient safety and medical errors in our nation's health care system, up to 98,000 Americans lose their lives each year as a result of preventable medical errors. $U_{p}$ to 7,000 die because of errors in prescribing medicine. And the cost of all these errors adds as much as $\$ 29$ billion to our medical bills.

(...) But too many families have been the victims of medical errors that are avoidable, mistakes that are preventable, tragedies, therefore, that are unacceptable ".
C'est pourquoi, il est suggéré à une faculté qui désire faire une réforme :

- considérant que les citoyens doivent être protégés par des examens de certification vérifiant les compétences professionnelles acquises ;

- considérant que parmi les éléments qui motivent les étudiants, partout et depuis toujours, les examens de certification jouent un rôle très important ;

- considérant que les étudiants sont supposés devenir professionnellement compétents grâce à (et plus souvent malgré) un programme de base ;

a) de construire un examen (ensemble d'outils d'évaluation dont on divise l'ensemble en 2 parties égales, l'une destinée à usage formatif et l'autre à usage certificatif) capable de mesurer, avec un niveau adéquat de validité, la performance de toutes les compétences professionnelles (dérivées de la réalité de la profession ou adaptées de catalogues existants ${ }^{18,19}$ );

b) de mettre ces outils à la libre disposition des étudiants sous forme d'évaluation formative ;

c) d'utiliser ces outils sous forme d'épreuve de certification pour vérifier le gain de compétence professionnelle des étudiants, entre leur entrée à l'université et la fin de leur cursus, leur donnant le droit de se présenter à un examen national éventuel et continuer en formation continue. Utiliser cette procédure tant que l'examen national ne sera pas au niveau d'objectifs correspondants à de réelles compétences professionnelles ;

d) de laisser aux enseignants la liberté individuelle d'enseigner « quoi » et " comment » selon leur choix ;

e) d'identifier des lieux d'observation et de pratique (pour des stages dans la communauté, dans des structures extra-hospitalières et dans des hôpitaux) où ont lieu des activités supervisées de soins (compétences professionnelles diagnostiques, thérapeutiques, de promotion de la santé, de prévention et/ou de réhabilitation, de recherche, etc.) ;

f) de laisser la liberté aux étudiants de suivre ou non l'enseignement proposé et le libre choix de lieux d'apprentissage compte tenu de leur performance lors des évaluations formatives et jusqu'à ce qu'ils aient démontré la maîtrise des compétences professionnelles identiques à celles de l'épreuve de certification.

Cela aide de prendre la précaution élémentaire de former une équipe cohérente concernant le choix et la mise en œuvre de l'orientation pédagogique pour former des médecins compétents. Et, certes, les « compétences pédagogiques » des enseignants en général mériteraient plus d'attention afin de donner à l'indispensable leader charismatique l'aide dont il aura besoin.

À votre bonne santé ... et avec modération. 


\section{Références}

1. Guilbert JJ. Guide pédagogique pour les personnels de santé. Genève: OMS, 1998 (Publication offset no 35, sixième édition), Genève : Organisation Mondiale de la Santé, 1998.

2. Jouquan J, Bail P. A quoi sengage-t-on en basculant du paradigme d'enseignement vers le paradigme d'apprentissage? Pédagogie Médicale 2003;4:163-75.

3. Guilbert JJ. Il est plus difficile de déménager un cimetière que de réussir une réforme des études médicales. Meducs 1993;4:2-3.

4. Guilbert JJ. Success or failure in change. Education for health 1996;9:189-200.

5. Guilbert JJ. Grandeur et décadence des réformes des études médicales. Enquête internationale. Meducs 1995;8:30-33.

6. Guilbert JJ. Curriculum change and strategies, past and present. Education for health 2001;14:367-72.

7. Baumann F. Tentatives de réformes dans l'enseignement des professions de la santé. Meducs 1992;3:2.

8. Lanares J. Maintenir et développer la qualité de l'enseignement. In : Un nouvel enseignement pour une nouvelle faculté de médecine. Université de Lausanne. MédecineđrHygiène (Numéro Spécial ; octobre), 2004:S8-S10.

9. Guilbert JJ. La Maladie des Profondeurs ou Ivresse des Profondeurs. (avec Commentaire de S. Abrahamson) Educaçao Médica, 1995;6:1 ;2-9.

10. Guba EG, Lincoln YS. Fourth Generation Evaluation. Newbury Park (CA): Sage Publications, 1989.

11. Guilbert JJ. Comment raisonnent les médecins. Réflexions sur la formation médicale. Genève: Editions Médecine et Hygiène, 1992.
12. Bussigel $M$, Barzansky BM, Grenholm $G G$. Innovation processes in medical education. New-York (NY): Praeger Publishers, 1988.

13. White KL. The task of medicine: Dialogue at Wickenburg. Menlo Park (CA): Henry J Kaiser Family foundation, 1988.

14. Abrahamson S. Diseases of the curriculum. J Med Educ 1978;53:951-7.

15. Rogers C. Freedom to learn. Colombus (OH): Merrill Publishing Company, 1969.

16. Miller GE. Teaching and Learning in Medical School revisited. In: Strom G, Walton H (Eds). Proceedings of the 1997 Conference of the Association for Medical Education in Europe: Innovations in Medical Education; 26-28 september 1976; Uppsala, Med Educ 1978;12 supplement:120-5.

17. Guilbert JJ. Réflexions personnelles sur une enquête comparant l'opinion des enseignants et des étudiants concernant le programme des études médicales en suisse. Meducs 1996;9:53-57.

18. Metz JCM, Stoelinga GBA, Pels Rijcken-Van Erp Taalman Kip EH, van den Brandt-Valkenburg BWM. Blueprint 1994, Training of Doctors in the Netherlands. Objectives of Undergraduate Medical Education. Nijmegen: University Publication Office 1994. Traduction anglaise de l'édition révisée 2001.[On-line] Disponible sur : http://www.imed.ac. at/lehre/pe_entwicklung/pol/blueprint.pdf

19. Bürgi H, Bader Ch, Bloch R et al. under a mandate of the Joint Commission of the Swiss Medical Schools. Swiss Catalogue of Learning Bobjectives for Undergraduate Medical Training. 2002. [On-line] Disponible sur : http://www.smifk.ch/

Manuscrit reçu le 13 juillet 2005; commentaires éditoriaux formulés à l'auteur le 30 novembre et le 28 décembre 2005; accepté pour publication le 3 janvier 2006. 